\title{
Exploring the Impact of Government Interventions on COVID-19 Pandemic Spread in Kuwait
}

Sana S. BuHamra, Kuwait University, Kuwait

Jehad Al Dallal, Gulf University for Science and Technology, Kuwait

\begin{abstract}
To model the trajectory of the pandemic in Kuwait from February 24, 2020 to February 28, 2021, the authors used two modeling procedures - auto regressive integrated moving average (ARIMA) with structural breaks and multivariate adaptive regression splines (MARS) — and then mapped the key breakpoints of the models to the set of government-enforced interventions. The MARS model, as opposed to the ARIMA model, provides a more precise interpretation of the intervention's effects. It demonstrates that partial and total lockdown interventions were highly effective in reducing the number of confirmed cases. When some interventions, such as enforcing regional curfews, closing workplaces, and imposing travel restrictions, were combined, their impact became significant. MARS method is recommended when exploring the impact of interventions on the spread of a disease. It does not require any prior assumptions about the statistical distribution of data, does not affect data collinearity, has simple and transparent functions, and allows for a more accurate analysis of intervention results.
\end{abstract}

\section{KEYWORDS}

ARIMA, COVID-19, Interrupted Time Series, Interventional Analysis, MARS Modeling, Precautionary Measures

\section{INTRODUCTION}

After the first novel coronavirus infection case was detected in Wuhan, China, in December 2019 (Zhu et al., 2020), the virus widely and rapidly spread across the world, leading the World Health Organization (WHO) to declare COVID-19 a pandemic on March 11, 2020 (Cucinotta \& Vanelli, 2020). Since then, the pandemic has become the most serious challenge for human societies and has negatively affected the world's healthcare and financial systems (McKibbin \& Fernando, 2020). By May 20, 2021, more than 165 million confirmed cases and 3.4 million deaths were reported globally (WHO Coronavirus Disease [COVID-19] Dashboard, 2021).

To slow down the spread of the virus and relieve health systems, health authorities adopted clinical practices, such as applying robust testing programs and, lately, speeding up vaccination. In addition, government authorities enforced physical nonpharmaceutical interventions (NPIs) and measures, such as mask-wearing, social distancing, quarantines, and lockdowns. However, applying these measures for long periods of time have social and economic consequences, such as high unemployment rates and bankruptcy (Policy Brief, 2020).

\section{DOI: 10.4018/IJHISI.288893}

This article published as an Open Access article distributed under the terms of the Creative Commons Attribution License (http://creativecommons.org/licenses/by/4.0/) which permits unrestricted use, distribution, and production in any medium, provided the author of the original work and original publication source are properly credited. 
Kuwait started to suffer from the COVID-19 pandemic after its first infection case was detected on February 24, 2020. Since then, the government of Kuwait has enforced several precautionary measures, including banning flights; closing workplaces, schools, and shopping malls; and applying total and partial lockdowns. As of May 20, 2021, Kuwait reported 295,861 confirmed infections, 281,920 recovered cases, and 1,711 deaths (WHO Coronavirus Disease [COVID-19] Dashboard, 2021).

Several studies have shown that interventions considered by health and government authorities are important and effective in controlling previous outbreaks (Leach, 2008; World Health Organization, Food and Agriculture Organization of the United Nations \& United Nations Children's Fund [UNICEF], 2012; Funk et al. 2017; Turner et al., 2020; Bernal et al., 2017). The interrupted time series (ITS) study design was widely and successfully used in some of these studies (Turner et al., 2020; Bernal et al., 2017). Several research studies used various statistical techniques to explore the impact of government precautionary measures on the spread of COVID-19 in different countries, such as China (Al-Rousan \& Al-Najjar, 2020), the UK (Ferguson et al., 2020), the US (Ferguson et al., 2020), Taiwan (Hsieh et al., 2020), Bahrain (Al Dallal et al., 2021), and Kuwait (Almeshal et al., 2020). These studies considered the pandemic at its early stage when its trajectory had a single wave. Therefore, it is not clear whether the same techniques are still applicable for analyzing current pandemic trajectories that contain multiple waves. In addition, the study of the case in Kuwait (Almeshal et al., 2020) only considered government precautionary measures enforced during the early pandemic days.

The present paper analyzes the impact of precautionary measures enforced since the beginning of the pandemic until February 28, 2021, on the spread of COVID-19 in Kuwait. During this period, the pandemic in Kuwait went through several subwaves. We used two modeling procedures: autoregressive integrated moving average (ARIMA) and multivariate adaptive regression splines (MARS). The models were constructed and compared, and the interpretation of their relationship to the precautionary measures were highlighted. The findings showed that some of the precautionary measures, such as complete lockdowns, were significantly effective in reducing the number of confirmed cases (referred to as "Cases" in the rest of this paper), thus containing the disease's spread. Other precautionary measures, such as partial lockdowns and travel restrictions, became effective only when they were combined. The findings showed that opening interventions caused the disease to resurface. Precautionary measures, such as a strict quarantine for arriving travelers, were found to be effective in preventing COVID-19's spread. The ARIMA model was found to be slightly better than the MARS model in terms of quality of fit measures. The results, however, showed that the latter model provided a more accurate interpretation of the interventions' effects.

The remainder of this paper is organized as follows. Section 2 provides an overview of the related work. Section 3 explains the applied methodology. Section 4 discusses the relationship between the trajectory of the pandemic, in terms of the number of cases, and the enforced interventions. Section 5 discusses the results of the two modeling procedures applied. Finally, in Section 6, we present the conclusions.

\section{RELATED WORK}

In this section, we review related literature on exploring the impact of precautionary measures on containing the spread of COVID-19. We provide an overview of the statistical techniques applied in this research.

\subsection{Literature Review}

Several studies investigated the effect of enforced precautionary measures on the spread of COVID-19. Al-Rousan and Al-Najjar (2020) applied time series forecasting models, including ARIMA, simple, Holt, and Brown, to model the pandemic in China and assess the impact of government interventions on containing the spread of COVID-19. The results showed that the growth rate for new infections 
decreased from $4.43 \%$ to $2.64 \%$ after applying the government interventions, and the growth rate for recovered cases increased from $3.86 \%$ to $4.91 \%$.

Ferguson et al. (2020) used models previously used to model the spread of influenza pandemic to explore the effect of several NPIs on reducing the level of demand on the healthcare system and the number of deaths caused by COVID-19 in the UK and the US. They found that the impact of NPIs on reducing the number of predicted deaths is limited when the NPIs are considered individually and significant when they are considered in combination. A study of the COVID-19 pandemic in Taiwan showed that the NPIs reduced the respiratory disease cases by $50 \%$ (Hsieh et al., 2020).

Al Dallal et al. (2021) proposed a methodology that considers the percentage of change in the values of the daily estimated reproduction number and the percentage of change in the number of performed tests before and after applying a precautionary measure. The methodology was considered to explore the impact of the precautionary measures applied and religious occasions celebrated in the Kingdom of Bahrain on the spread of COVID-19 pandemic. The results showed that most of the enforced precautionary measures were effective in reducing the spread of the disease by a percentage ranging from $20.2 \%$ to $41.8 \%$, whereas a religious occasion involving large gatherings caused the spread of the disease to increase by $28.7 \%$.

Almeshal et al. (2020) used compartmental and logistic regression models to predict the pandemic's future trajectory in Kuwait and to assess the effectiveness of the Kuwait government's interventions on containing the spread of the virus for a relatively short period of time from February 24,2020 , to April 19, 2020. To study the impact of an intervention, the authors applied their prediction models with and without considering the cases reported after the intervention. They also compared the prediction results in terms of the estimated pandemic size and end dates.

Vokó and Pitter (2020) used the ITS analysis to explore the impact of social distance measures implemented in 28 European countries on the spread of COVID-19. The results proved that social distancing meaningfully contributed to containing the spread of COVID-19 in Europe.

\subsection{Applied Statistical Techniques}

In this section, we provide a brief overview of some of the statistical methods on which our proposed models are based.

\subsubsection{ARIMA Models}

ARIMA is a model used to understand past data or predict future data in a time series (Tandon et al., 2020). The response variable $Y_{t}$ is measured at regular intervals (minute, hour, day, month, etc.). A univariate time series uses only previous values in times $\left(\mathrm{Y}_{\mathrm{t}-\mathrm{i}}\right)$ to predict future values, whereas the series is a multivariate when other predictors are included in the model. The term "autoregression" (AR) refers to a model that regresses the dependent variable on its own lagged or past values. The difference between raw observations is represented by integrated (I). To make it stationary, data values are replaced by the difference between the data values and the previous values. A stationary model demonstrates that the data are consistent over time. The moving average (MA) denotes that forecasting errors are linear functions of previous errors.

$\operatorname{An} \operatorname{ARIMA}(\mathrm{p}, \mathrm{d}, \mathrm{q})$ is a standard notation for a nonseasonal ARIMA, where $\mathrm{p}$ (lag order) is the number of lag observations, $d$ is the number of times that the raw observations are differenced, and $\mathrm{q}$ is the order of the MA. The steps for fitting an ARIMA model to a (nonseasonal) time series data (PennState, 2021) are as follows: Identify any outliers by plotting the data first and then using a Box-Cox transformation to stabilize the variance if necessary. The Box-Cox method considers a family of transformations on a strictly positive response variable and is defined as 


$$
g_{\lambda}(y)= \begin{cases}\frac{y^{\lambda}-1}{\lambda} & \lambda \neq 0 \\ \log (y) & \lambda=0\end{cases}
$$

The $\lambda$ parameter is chosen by maximizing the log-likelihood,

$$
L(\lambda)=-\frac{n}{2} \log \left(R S S_{\lambda} / n\right)+(\lambda-1) \sum \log \left(y_{i}\right)
$$

where RSS is the residual sum of squares and log is the natural logarithm.

If the time series is nonstationary, take the first differences and repeat differencing as needed until the data become stationary. The autocorrelation function (ACF) and partial autocorrelation function (PACF) plots are used to examine the series' residual time errors. Out of the appropriate models found, select the best model based on the Akaike information criterion (AIC). The final model can be used for forecasting. The "auto.arima" procedure does not cover all of these steps, and a manual check must therefore be considered. For a seasonal time series, the data should be corrected to reflect the differences between the seasons.

\subsubsection{MARS Algorithm}

The MARS algorithm is a nonlinear and nonparametric regression model. It was first introduced by Friedman (1991). This model estimates the relationships between the dependent and independent variables using piecewise linear splines. The power of the MARS model over other competitive models has been addressed in various publications (Boehmke \& Greenwell, 2019; Lee et al., 2006; OEB, 2017). Traditional statistical analyses, such as mixed linear models, struggle to perform well when the number of possible predictors is extremely high. The MARS model is formulated as

$$
y_{t}=f\left(x_{t}\right)=\beta_{0}+\sum_{i=1}^{k} \beta_{i} B_{i}\left(x_{t}\right)+\varepsilon \text {, }
$$

where $\beta_{0}$ is a constant representing the model intercept, $\mathrm{B}_{\mathrm{i}}\left(\mathrm{x}_{\mathrm{t}}\right)$ is a basis function $(\mathrm{BF}$, spline function), $\mathrm{k}$ is the number of BFs, $\beta_{\mathrm{i}}$ is the constant coefficient of the ith $\mathrm{BF}$, and $\varepsilon$ is the unexplained variation.

MARS generates knots or cut points for the different variables in the model. The algorithm searches through all possible knot locations and through all variables and their interactions. It accomplishes this through the use of variable combinations known as BFs (spline functions). MARS builds the model with these BFs. Each BF in MARS models consists of a constant (i.e., the intercept) and a hinge function, as well as a product of two or more hinge functions, to model variable interactions if they exist. Hinge functions are an important part of MARS models and take the form of $\max (0$, $\mathrm{x}-\mathrm{c})$ or $\max (0, \mathrm{c}-\mathrm{x})$, where $\mathrm{c}$ is a constant called a knot (i.e., a breakpoint value) and $\max (0, \mathrm{x}-\mathrm{c})$ and $\max (\mathrm{x}-\mathrm{c}, 0)$ refer to hinge functions where $\max (0, \mathrm{x}-\mathrm{c})$ is 0 if $\mathrm{x}-\mathrm{c}<0$, else $\mathrm{x}-\mathrm{c}$.

The MARS algorithm can be summarized as follows. It begins by locating a single point (knot) $c$ across a spectrum of $x_{t}$ values, where two separate linear relationships between $y_{t}$ and $x_{t}$ achieve the lowest error (i.e., smallest error sum of squares [SSE]). Consequently, a BF is developed. MARS continues until many knots are discovered using the forward stepwise algorithm, which results in an overestimated model with a large number of BFs. Although many knots would match a good relationship with the training data, it would not be good at predicting new observations. As a result, 
once the entire set of knots has been found, MARS employs a backward stepwise algorithm to eliminate knots that do not significantly contribute to predictive accuracy. Cross-validation is used to find the optimum number of knots in this operation, which is known as "pruning." Based on the SSE criterion, BFs are extracted one by one from the overfit model during the pruning stage. After each deletion, the model is refitted, and each reduced model is evaluated using the generalized cross-validation (GCV) criterion, which is a weighted mean squared error (MSE) criterion used to avoid overfitting. A model with a GCV R2 score near 1 is considered good, while one with a GCV R2 score near zero is considered poor. The GCV R2 of an optimal model is maximized. MARS can also multiply modeling parameters to include variable interaction terms. This is the second tuning parameter and is measured in terms of degrees of freedom, abbreviated as "degree." MARS can generally only add parameters to the model if they increase the model's GCV value.

\subsubsection{Quality of Fit Criterion}

When models are built, specific criteria must be used to determine and choose the best among them. Models can be used to describe relationships and forecast outcomes in general. It is preferable to use a small and interpretable model to describe relationships. If the aim is to make predictions, models with the fewest errors and no overfitting are preferred.

The coefficient of determination $\left(\mathrm{R}^{2}\right)$ and MSE, two well-known metrics for determining the model fit, have a drawback in that their values do not worsen when an additional variable is applied to the model even though that predictor has no significance to the model. Therefore, if we just consider these measures, we will still choose the largest model, and our fitting will lead to noise. Since it is often better to have a small model with a good fit, we must follow other model criteria that consider the size of the model (i.e., model terms) even if we sacrifice a small amount of quality-of-fit in exchange for having a small model. Following that, we'll go through some quality criteria that consider the model's size. Extensive statistical explanations of these criteria are given in Dalpiaz (2020). With the exception of adjusted R-squared (Adj. $\mathrm{R}^{2}$ ), the smaller the criterion value, the better for model selection.

Akaike information criterion (AIC): This criterion is a measure of the quality of the model and is defined as $2 \log$ (maximum log-likelihood of a regression model).

Bayesian information criterion (BIC): Just like AIC, the BIC quantifies the balance between a model that fits well and the number of model parameters. However, it usually selects a smaller model than AIC for a reasonable sample size.

Adjusted R-squared (Adj. $\left.\mathrm{R}^{2}\right)$ : It is a modified coefficient of determination $\left(\mathrm{R}^{2}\right)$ that has been adjusted for the number of predictors in the model. It can decrease as predictors are added.

Cross-validation criterion $(\mathrm{CV})$ : The idea is to create a train/test split of the data, including some sample observations not included in the model-building process and will be used to test the performance of the fully trained model. It provides a prediction error estimate.

Cross-validated RMSE: It is mainly used to measure the model's performance during crossvalidation by means of a root mean squared error (RMSE). The RMSE is a measure of the model's average prediction error when predicting the outcome of an observation.

Mean absolute error (MAE): It is an alternative to the RMSE that is less sensitive to outliers. It corresponds to the average absolute difference between observed and predicted outcomes. During cross-validation, MAE is also used to assess model performance.

\section{METHODOLOGY}

\subsection{The Data}

We collected COVID-19 data for Kuwait from the official website of the Ministry of Health of Kuwait (Ministry of Health, Kuwait, 2021). The reported daily data, including Cases, recovered cases, deaths, ICU patients, and number of performed PCR tests. In this research, we are interested in the daily 
number of cases since the first case was reported on February 24, 2020, until February 28, 2021, when we collected the data. We estimated the positivity rate as 100 times the ratio of daily new cases to daily PCR tests for each of the dates studied. It is an important metric because it indicates how widespread the infection is in the area where testing is taking place and whether testing levels are keeping up with disease transmission levels (Dowdy \& D’Souza, 2020).

\subsection{Adopted Interventions and Major Related Events in Kuwait}

Government and health authorities in Kuwait enforced several precautionary measures to slow down the spread of COVID-19 in Kuwait. The major ones are summarized in Table 1. These measures started by enforcing travel restrictions (E1) on March 7, 2020, followed by a series of graduating lockdown restrictions, including E2, E3, and E4, and ending with a total lockdown (E6) enforced on May 10, 2020 , for three weeks. These restrictions started to be relaxed by a series of reopening interventions, including E7, E8, and E9, which were followed by a complete termination of the lockdown (E10) on August 30, 2020, and a partial resumption of the commercial flights (E11 and E13). By the end of December 2020, Kuwait started vaccinating people with two approved vaccines, including Pfizer/ BioNTech and Oxford/AstraZeneca. When the second main pandemic wave started at the beginning of February 2021, the government resumed some partial lockdown decisions, including E14 and E15.

\subsection{COVID-19 Interventional Study Models Proposed}

Our objective in this work was to investigate and quantify the effects of nonpharmaceutical interventions (Table 1) implemented in Kuwait during the pandemic period under consideration. Two modeling procedures were used to achieve this goal. The first model was based on ARIMA, which displayed the time series' greatest breakpoints with a structural fit (Garziano, 2017). This model was constructed in three major steps. First, we applied structural change analytics to the original time series data. Throughout this analysis, the main breakpoints and their respective break dates were identified. The minimum BIC was used to select the optimal number of breakpoints $\mathrm{m}$. Second, we plotted the original time series against the structural breaks and the associated confidence intervals. Third, the fitted multiple-level changes of the structural breaks could be used as an intervention component in the ARIMA model. The final version of the model must go through a residual analysis to ensure that the stationary and other model assumptions were satisfied.

The MARS model, including both quantitative (day) and qualitative (interventions) predictors, was used in the second method. The BFs of the mixed model were expressed differently for each type of variable. The two modeling procedures were compared using RMSE, MAE, and result interpretability. In all of our analyses, we used $\mathrm{R}$ version 4.0.2, 2020.

\section{INVESTIGATING CASES IN TIME VS. INTERVENTIONS}

The progression of the pandemic, as measured by the daily number of cases (positive PCR test), is graphically depicted in Figure 1, as well as the activation of the 14 interventions mentioned in Table 1 from February 24, 2020, to February 28, 2021. Table 2 shows the number of days that each intervention was absent or active during the study period. E1 was triggered at a very early stage of the pandemic and is still active, so its effect is likely to be masked by subsequent interventions. Even though interventions E13-E14 were initiated at the end of the study period, there is insufficient evidence to allow a reliable comparison of their effects afterward.

The time series of Cases by E2 (chain of lockdown interventions) and by E4 (regional ban) are very similar in terms of activation periods (Figure 1). Therefore, we performed a chi-square test of independence between the two events E2 and E4. The resulting chi-square of 219.843 (df=1, p-value $<0.001$ ) is highly significant, and the estimated effect size (Cramér's V) $=0.77$ is also very high. Cramér's V is an effect size measurement for the chi-square test of independence. It assesses how 
Table 1. Major government precautionary measures

\begin{tabular}{|c|c|c|c|}
\hline $\begin{array}{l}\text { Effective } \\
\text { Date }\end{array}$ & Code & Intervention Description & Status/Ended \\
\hline 7-Mar-20 & E1 & Outbound and inbound flights are banned for certain affected countries. & Ongoing* \\
\hline 12-Mar-20 & E2 & $\begin{array}{l}\text { Chain of interventions: Vacation for employees. No gatherings in } \\
\text { restaurants and coffeehouse. Closing mosques, shopping malls, and gyms. } \\
\text { Banks closed for } 2 \text { weeks. Closing Kuwait stock market. }\end{array}$ & 30-Jun-20 \\
\hline 22-Mar-20 & E3 & Partial curfew: $5 \mathrm{PM}$ to $4 \mathrm{AM}$ & 9-May-20 \\
\hline 6-Apr-20 & E4 & Applying regional total ban & 9-Jul-20 \\
\hline 19-Apr-20 & E5 & $\begin{array}{l}\text { Evacuating citizens who are outside of Kuwait until May 6, which } \\
\text { includes } 500,000 \text { Kuwaiti citizens }\end{array}$ & 6-May-20 \\
\hline 10-May-20 & E6 & Lockdown (total ban, stopping the delivery of orders) & 30-May-20 \\
\hline 31-May-20 & E7 & $\begin{array}{l}\text { Total lockdown switched to a partial curfew for } 12 \text { hours, resume online } \\
\text { ordering and delivery, more regions added to regional ban }\end{array}$ & 30-Aug-2020 \\
\hline 10-Jun-20 & E8 & Permission to pray in mosques while maintaining health regulations & Ongoing* \\
\hline 30-Jun-20 & E9 & Partial opening for workplaces and malls with certain criteria & 29-Aug-20 \\
\hline 30-Aug-20 & E10 & $\begin{array}{l}\text { Terminating partial curfew and resuming normal life but limited to } \\
\text { banning some commercial activities and gathering including making } \\
\text { feasts gathering and weddings }\end{array}$ & 7-Mar-21 \\
\hline $1-$ Oct-20 & E11 & Resuming some commercial flights from and to Kuwait & Ongoing* \\
\hline 24-Dec-20 & E12 & Starting vaccinating people & Ongoing* \\
\hline $17-J a n-21$ & E13 & $\begin{array}{l}\text { Allowing housekeepers to fly to Kuwait under certain regulations } \\
\text { including quarantine for two weeks and presenting negative PCR result. }\end{array}$ & Ongoing* \\
\hline 7-Feb-21 & E14 & $\begin{array}{l}\text { All commercial activities are banned from 8:00 PM until 5:00 AM except } \\
\text { Co-ops and pharmacies - Gyms and Spas shops closed completely }\end{array}$ & 7-Mar-21 \\
\hline
\end{tabular}

*By the end of the study, it was still ongoing.

strongly two categorical variables are associated (Sheskin, 2011). These results most likely indicate a confounding effect in a sense we will not be able to distinguish between the effects of the two events.

In a similar way, the test of independence for E2 and E3 (partial curfew) is significant (chisquare $=128.418$ with $\mathrm{df}=1, \mathrm{p}$-value $<0.001$ ), and the estimated effect size (Cramér's $\mathrm{V}$ ) is 0.588 , indicating a strong association between these two events. In addition, E3 and E4 are strongly associated (chi-square $=54.19$, with $\mathrm{df}=1$, p-value $<0.001$, Cramér's $\mathrm{V}=0.382$ ). Overall, it appears that each of the three events E2, E3, and E4 has an impact that is mixed (confounded) with the effects of the other two.

It is also noticeable from Figure 4 that after the total ban (E6), the curve obviously decreased. The two interventions, E5 (citizens' evacuation from abroad) and E6 (total ban), were found to have no relationship (chi-square $=0.0294$, with $\mathrm{df}=1, \mathrm{p}$-value $=0.587$ ). Also, E7 (partial curfew) and E8 (allowing mosques prayer with precautions) were found to have a very weak relationship (chisquare $=4.17$, with $\mathrm{df}=1$, p-value $=0.041$, Cramér's $\mathrm{V}=0.106$ ).

The progression of Cases remained relatively low compared to those lately reported until the E11 (resuming commercial flights) after the partial opening (E9) and the termination of the partial curfew (E10), which results in returning to a normal life. After these interventions, the second wave of the pandemic has begun to spread. It is found that E10 and E11 are strongly associated (chisquare $=258.191$, with $\mathrm{df}=1$, p-value $<0.001$, Cramér's $\mathrm{V}=0.834$ ). This most likely means that we won't be able to tell the difference between the two events' outcomes.

The vaccination campaign (E12) began in late December 2020, but the curve continued to rise two months later until the end of this study period. 
Figure 1. Time series of cases vs. interventions (o days absent, $\Delta$ days active)

E1

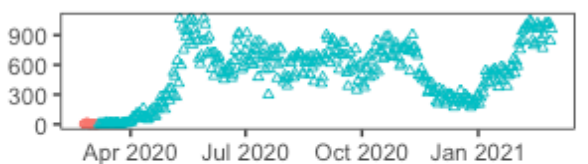

E3

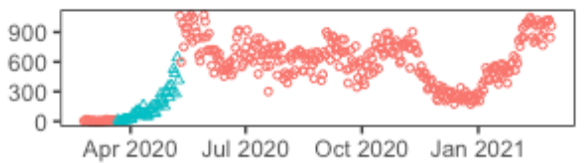

E5

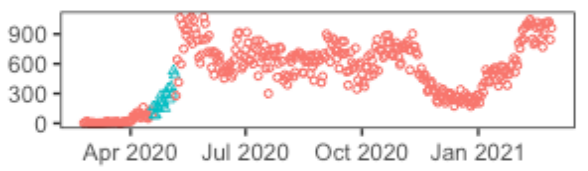

E7

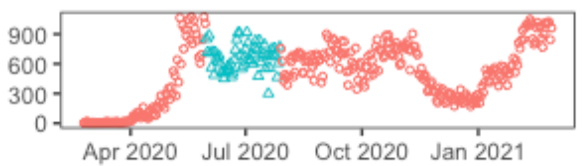

E9

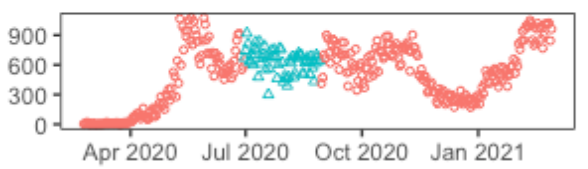

E11

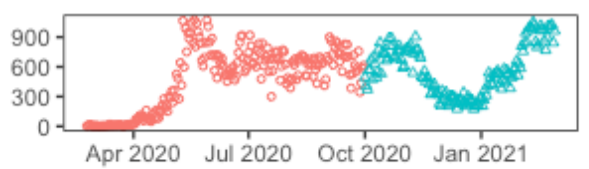

E13

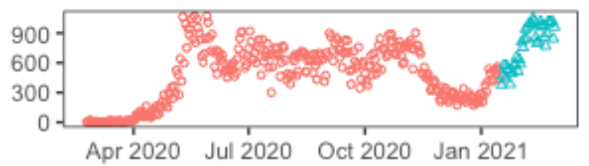

E2

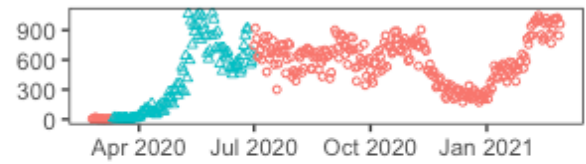

E4

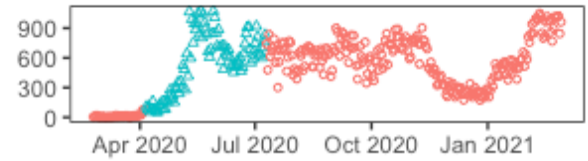

E6

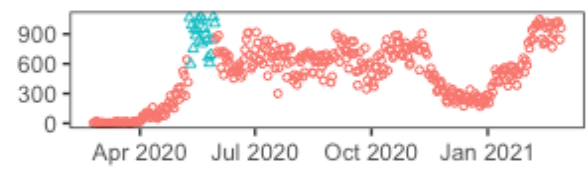

E8

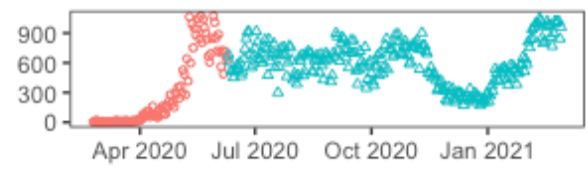

E10

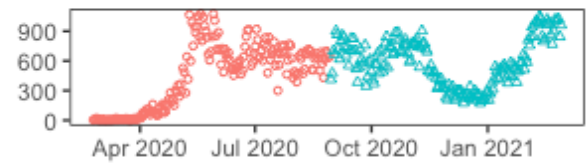

E12

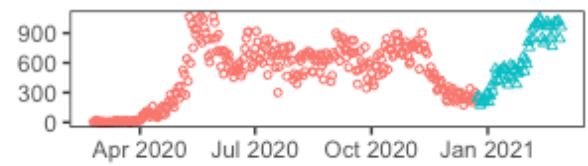

E14

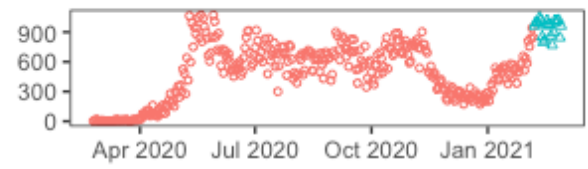

Figure 2 shows the data distribution by the most recent interventions in this study, E13 and E14. The second wave of the pandemic, as well as the activation of E13, began in January 2021. Before and after this period, the mean and median have risen significantly. When comparing cases before and after January 21, the mean increased from 479.9 to 778.0, and the median increased from 526.5 to 840.0. Similarly, when comparing cases before and after February 21, the mean increased from 487.2 to 946.2 , and the median increased from 530.0 to 977.5 cases.

Table 3 provides overall descriptive measures for the data from February 24, 2020, to February 28, 2021. The median number of daily cases is 553 , which is greater than the mean of 514.4 because the 
Table 2. Intervention frequency table E1-E14

\begin{tabular}{|l|l|l|l|l|l|l|l|l|l|l|l|l|l|l|}
\hline Events & E1 & E2 & E3 & E4 & E5 & E6 & E7 & E8 & E9 & E10 & E11 & E12 & E13 & E14 \\
\hline Absent (0) & 12 & 260 & 322 & 276 & 353 & 350 & 312 & 107 & 310 & 188 & 220 & 304 & 328 & 349 \\
\hline Active (1) & 359 & 111 & 49 & 95 & 18 & 21 & 59 & 264 & 61 & 183 & 151 & 67 & 43 & 22 \\
\hline
\end{tabular}

Figure 2. Cases by E13 and E14
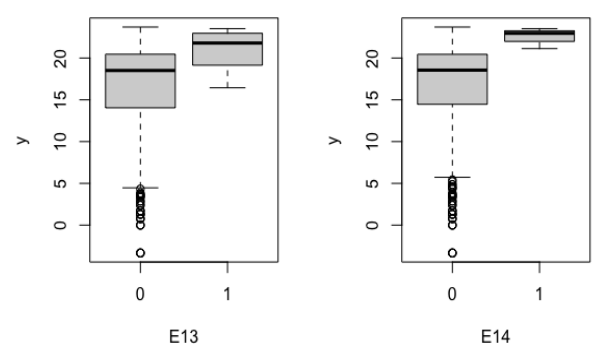

Table 3. Summary Statistics of Cases and Positivity Rate

\begin{tabular}{|l|l|l|l|l|l|l|}
\hline Variable & $\mathbf{N}$ & $\begin{array}{l}\text { Min } \\
\text { Max }\end{array}$ & $\begin{array}{l}\text { Q1 } \\
\mathbf{Q 3}\end{array}$ & median & Mean (sd) & 95\% Cl \\
\hline Cases & 371 & $\begin{array}{l}0 \\
1073\end{array}$ & $\begin{array}{l}284.5 \\
732\end{array}$ & 553 & $514.4(293)$ & $(484.5,544.3)$ \\
\hline $\begin{array}{l}\text { Positivity } \\
\text { rate }\end{array}$ & 275 & $\begin{array}{l}2.8 \\
27.7\end{array}$ & $\begin{array}{l}7.8 \\
17.4\end{array}$ & 12.96 & $12.84(5.77)$ & $(12.2,13.5)$ \\
\hline
\end{tabular}

latter is sensitive to very small values at the start of the series. The median positivity rate is $12.96 \%$, which is higher than the recommended threshold of 5\%. The WHO recommended in May 2020 that the positivity rate remain below $5 \%$ for at least two weeks before governments consider reopening. If we are successful in controlling coronavirus transmission, this threshold may be lowered over time to loosen social restrictions even more and allow for very large gatherings or meetings of people traveling from all over the world (Dowdy \& D'Souza, 2020). Figure 3 depicts the positivity rate beginning on May 30, 2020, when Kuwaiti officials started announcing the number of daily PCR tests.

\section{INTERVENTION ANALYSIS}

In this section, we used nominal predictors, the interventions listed in Table 1, and day number as a time trend to fit Y (Cases). The goals were to determine which NPIs have a significant effect on the curve of cases and to quantify this effect.

\subsection{Intervention Analysis Using Arima Models}

A Box-Cox transformation with an optimal $\lambda=0.3$ was applied to the response variable of interest (Cases) in our modeling procedures in this section and in the following section. This transformation 
Figure 3. Positivity rate by month

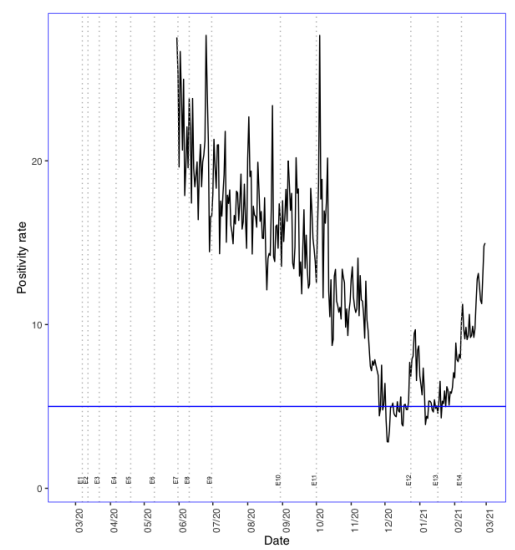

Figure 4 Plot of breakpoints against BIC and RSS

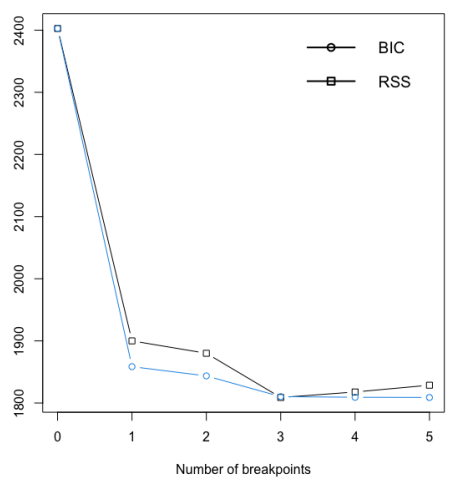

resulted in obtaining Cases $^{t}$ values. The goal of this transformation was to stabilize the time series' nonhomogeneity of variance and make the pattern more consistent across the entire data set. Simpler patterns tend to produce more accurate forecasts.

The best breaks at $\mathrm{m}=3$ is achieved with minimum $\mathrm{BIC}$ value $=1,809$, as shown in Figure 4 where $\mathrm{BIC}$ is presented on the left $\mathrm{y}$-axis and RSS on the right $\mathrm{y}$-axis. The plot of the original time series against its structural breaks and their confidence intervals is given in Figure 5. It can be observed that there are three major changes on the structural level on April 23, 2020, November 10, 2020, and January 4, 2021.

Table 4 presents the optimal breakpoints represented in terms of day number and corresponding break dates in addition to $95 \%$ confidence interval (CI). The value for $m=1$ corresponds to the first breakpoint, which occurs at observation number 60 (on April 23, 2020), with confidence interval ranges between observation numbers 59 and 61. It was similar for the second and third breakpoints, as shown in the table. 
Figure 5. Plot of actual cases ${ }^{t}$ values versus its structural breaks (breakpts)

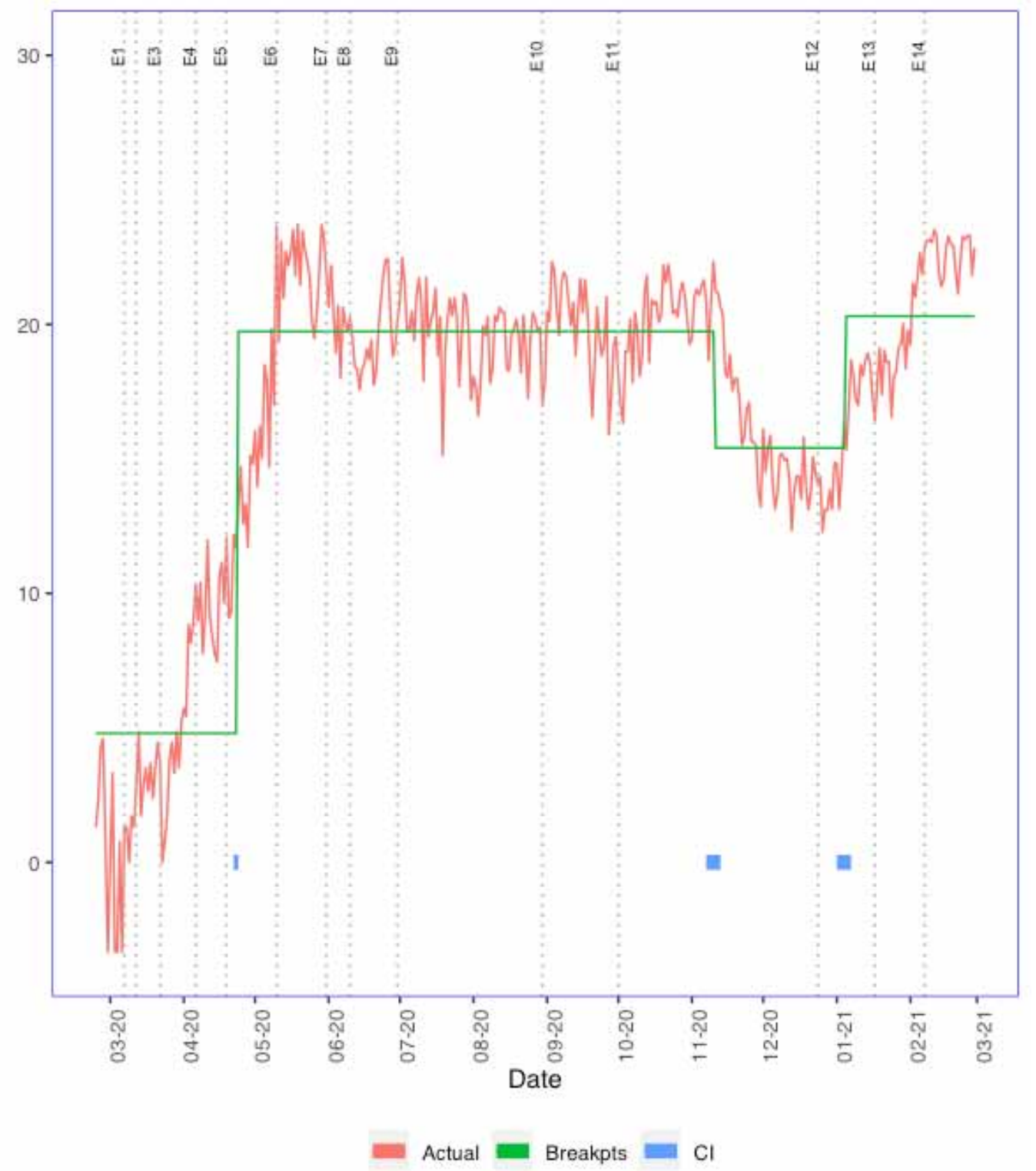

To translate the effect of the structural breaks on the original time series, we performed a backtransformation of the Box-Cox transform and calculated the difference in means between the cases before and after a breakpoint using the Welch two sample t-test. The mean difference $\left(\mu_{\mathrm{d}}\right)$ and its $95 \% \mathrm{CI}$ corresponding to each of the three breakpoints are as follows. A mean difference of $\mu_{\mathrm{d}}=611.7$ indicated an average increase of 612 cases after the first breakpoint on April 23, 2020, with 95\% CI of mean difference between $(583.9,639.4)$ cases. An average decrease of about 318 cases $\left(\mu_{d}=-318.3\right)$ occurred after the second breakpoint on November 11, 2020, with 95\% CI of mean difference between (-364.7, -271.9). Lastly, an average increase of 378 cases $\left(\mu_{d}=378.2\right)$ after the third breakpoint on January 4 , 2021, with $95 \%$ CI of mean difference between $(305.4,451.0)$. 
Table 4 . Breakpoints of cases ${ }^{t}$ time series

\begin{tabular}{|l|l|l|l|l|}
\hline \multirow{2}{*}{} & \multicolumn{2}{|l}{ Breakpoints at observation No. with 95\% CI } & Ureakpoint \\
\cline { 2 - 5 } & $\mathbf{m}$ & Lower 2.5\% & $\begin{array}{l}60 \\
\text { (April 23, 2020) }\end{array}$ & $\begin{array}{l}61 \\
\text { (April 24, 2020) }\end{array}$ \\
\hline $\begin{array}{l}\text { Breakpoint } \\
\text { (Break date) }\end{array}$ & 1 & $\begin{array}{l}59 \\
\text { April 22, 2020) }\end{array}$ & $\begin{array}{l}261 \\
\text { (Nov. 10, 2020) }\end{array}$ & $\begin{array}{l}264 \\
\text { (Nov. 11, 2020) }\end{array}$ \\
\hline $\begin{array}{l}\text { Breakpoint } \\
\text { (Break date) }\end{array}$ & 2 & $\begin{array}{l}258 \\
\text { (Nov. 9, 2020) }\end{array}$ & $\begin{array}{l}316 \\
\text { (Jan. 4, 2021) }\end{array}$ & $\begin{array}{l}319 \\
\text { (Jan. 7, 2021) }\end{array}$ \\
\hline $\begin{array}{l}\text { Breakpoint } \\
\text { (Break date) }\end{array}$ & 3 & $\begin{array}{l}313 \\
\text { (Jan. 1, 2021) }\end{array}$ & \multicolumn{2}{|l}{} \\
\hline
\end{tabular}

Notably, the first breakpoint occurred after applying a set of lockdown interventions, including E1-E4. The breakpoint is observed after about two weeks from applying the total ban intervention (E4) for regions, including crowded housing for workers, where implementing strict social distancing measures is potentially difficult. The decrease in the number of cases observed at the second breakpoint is potentially due to the effectiveness of the preceding complete lockdown intervention (E6), which was followed by a set of partial opening interventions (i.e., E7-E11) accompanied with strict precautionary measures. The decline in the number of cases proved that these precautionary measures were successfully implemented. Finally, the third breakpoint observed on January 4, 2021, showing an increase in the number of cases, occurred after the coronavirus UK variant (B.1.1.7) globally spread. The breakpoint potentially showed that this coronavirus variant significantly affected Kuwait at that time.

The fitted multiple levels (breaks in Figure 5) can be used as an intervention variable to fit an ARIMA model. This variable was inserted into the Arima() function in $\mathrm{R}$ as an xreg argument (sfit as a predictor). Next, we searched for the best ARIMA model. The auto.arima function in R suggested the model Arima $(1,1,4)$. Nevertheless, the residual analysis of this model did not reveal white noise residuals, indicating that this model does not capture all the structures of the original time series. Therefore, we performed a manual search for the best model based on the quality-of-fit criteria in addition to the model residual analysis using ACF/PACF plots. The results are listed in Table 5.

Table 5. Comparison of different ARIMA models of cases $^{t}$

\begin{tabular}{|l|l|l|l|}
\hline \multirow{2}{*}{$\begin{array}{l}\text { Model } \\
\text { ARIMA(p,d,q) }\end{array}$} & Criteria & BIC \\
\cline { 2 - 4 } & AIC & AICc & 1351.3 \\
\hline Model_1: ARIMA(5,1,2) & 1316.1 & 1316.2 & 1357.2 \\
\hline Model_2: ARIMA(5,1,3) & 1318.1 & 1318.7 & 1350.9 \\
\hline Model_3: ARIMA(4,1,2) & 1319.6 & 1320.0 & \\
\hline
\end{tabular}

Model_1 is the top candidate since it provides the best AIC and AICc metrics. Using the checkresiduals() function within the forecast package and the sarima() within the astsa package, the assumptions of Model_1 were examined and presented in Figure 6. We also tested the autocorrelation of the residuals using the Ljung-Box test within the FitARMA package. The desirable result is to have a nonsignificant ( $\mathrm{p}$-value $>0.05$ ) test. That was achieved for Model_1 with test values $\mathrm{Q}^{*}=64.6$, $\mathrm{df}=66$, and $\mathrm{p}$-value $=0.5258$, as anticipated. Hence, the predicted COVID-19 model $(\operatorname{ARIMA}(5,1,2)$ for Cases $^{t}$ with a structural fit) is represented as 
Cases $^{\mathrm{t}}=0.64$ ar1 $-0.61 \operatorname{ar} 2-0.29 \operatorname{ar} 3-0.18$ ar4 -0.14 ar5 $-1.21 \mathrm{ma} 1+0.94 \mathrm{ma} 2+0.15$ sfit, where the response variable Cases ${ }^{t}$ is the Cox-Box transformation of Cases. The terms (ar1, ar2, $\ldots, \operatorname{ar} 5),(\mathrm{ma} 1, \mathrm{ma} 2)$, and sfit terms in the model represent the five autoregressive terms, the two MA terms, and the structural break fit, respectively. All the model coefficients were tested and found to be significant. The model RMSE $=1.39$ and $\mathrm{MAE}=1.03$.

\subsection{Intervention Analysis Using MARS}

Four models were built as part of our MARS modeling procedure. The first involved using binary interventions E2-E14 in addition to a quantitative variable "day" as predictors. The second MARS model used day and curfew as predictors. In this model, we combined the lockdown interventions (E3, E6, and E7) into a single binary intervention called curfew because they all referred to a lockdown but at different times. In the third MARS model, curfew was represented as a quantitative variable in terms of hours of lockdown. The final model quantified E4, the regional ban, as occupancy (percent) in the total shutdown areas. The last two models produced the same results. The second model with predictors (day, E2, E4, E5, E8, E9, E10, E11, E12, E13, E14, and curfew) was chosen and is presented in this section.

When building the MARS models, we applied the forward and backward steps explained in Section 2.2.2. We found that the best MARS model for COVID-19 data has 12 additive BFs. The best cross-validated RMSE and MAE values are 1.48 and 1.17, respectively.

Figure 6 . Residual analysis plots for the $\operatorname{ARIMA}(5,1,2)$ with structural breaks
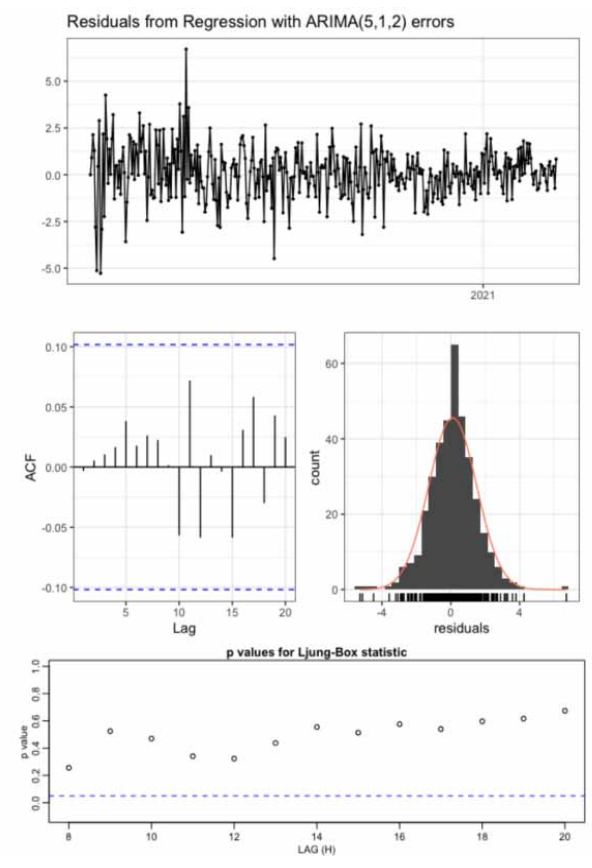

Our optimal model has 13 terms (with intercept) based on four predictors, as shown in Figure 7. Any additional terms retained in the model, over and above the optimal terms, resulted in less than 0.001 improvement in the GCV R2 (GRSq). Day, curfew, E5, and E9 were the four predictors chosen to fit the best model for Casest. 


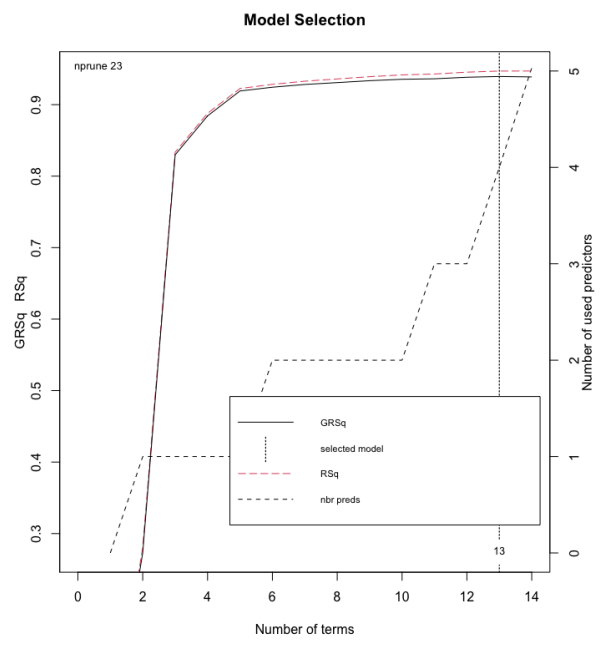

Table 6 explicitly presents the selected BFs with the most relevant variables contributed as additive terms in the model. The first three BFs correspond to binary interventions E5, E9, and curfew when activated. The remaining nine BFs involve breakpoints or knots of the time trend at days 12 (March 6, 2020), 84 (May 17, 2020), 108 (June 10, 2020), 204 (September 14, 2020), 216 (September 26, 2020), 246 (October 26, 2020), 306 (December 25, 2020), and 318 (January 6, 2021). The final model is expressed as a linear combination of the BFs as follows:

Cases $^{\mathrm{t}}=-23.406-1.737 \mathrm{BF}_{1}+1.186 \mathrm{BF}_{2}-3.071 \mathrm{BF}_{3}+0.684 \mathrm{BF}_{4}+0.312 \mathrm{BF}_{5}-0.812 \mathrm{BF}_{6}$ $+0.106 \mathrm{BF}_{7}-0.182 \mathrm{BF}_{8}+0.325 \mathrm{BF}_{9}-0.273 \mathrm{BF}_{10}+0.515 \mathrm{BF}_{11}-0.226 \mathrm{BF}_{12}$.

Table 6. Model coefficients and BFs

\begin{tabular}{|l|l|l|}
\hline Model term & Coeff. & Basis functions \\
\hline Intercept & -23.406 & \\
\hline $\mathrm{E}_{1}^{\S}$ & -1.737 & $\mathrm{BF}_{1}=\mathrm{I}(\mathrm{E} 5=1)^{\S \S}$ \\
\hline $\mathrm{E}_{1}^{\S}$ & 1.186 & $\mathrm{BF}_{2}=\mathrm{I}(\mathrm{E} 9=1)^{\S \S}$ \\
\hline Curfew $_{1}^{\S}$ & -3.071 & $\mathrm{BF}_{3}=\mathrm{I}(\mathrm{Curfew}=1)^{\S \S}$ \\
\hline $\mathrm{h}($ Day-12) & 0.684 & $\mathrm{BF}_{4}=\max (0$, Day-12) \\
\hline $\mathrm{h}(84-$ Day $)$ & 0.312 & $\mathrm{BF}_{5}=\max (0,84-$ Day $)$ \\
\hline $\mathrm{h}($ Day-84) & -0.812 & $\mathrm{BF}_{6}=\max (0$, Day-84 $)$ \\
\hline $\mathrm{h}($ Day-108) & 0.106 & $\mathrm{BF}_{7}=\max (0$, Day-108 $)$ \\
\hline $\mathrm{h}($ Day-204) & -0.182 & $\mathrm{BF}_{8}=\max (0$, Day-204 $)$ \\
\hline $\mathrm{h}($ Day-216) & 0.325 & $\mathrm{BF}_{9}=\max (0$, Day-216 $)$ \\
\hline $\mathrm{h}($ Day-246) & -0.273 & $\mathrm{BF}_{10}=\max (0$, Day-246 $)$ \\
\hline $\mathrm{h}($ Day-306) & 0.515 & $\mathrm{BF}_{11}=\max (0$, Day-306 $)$ \\
\hline $\mathrm{h}($ Day-318) & -0.226 & $\mathrm{BF}_{12}=\max (0$, Day-318 $)$ \\
\hline
\end{tabular}

Sintervention at level 1 (Active); $\$ \$$ Is an indicator function, I = 1, if condition (.) is satisfied 
Figure 8. Actual cases ${ }^{t}$ time series against its MARS fit

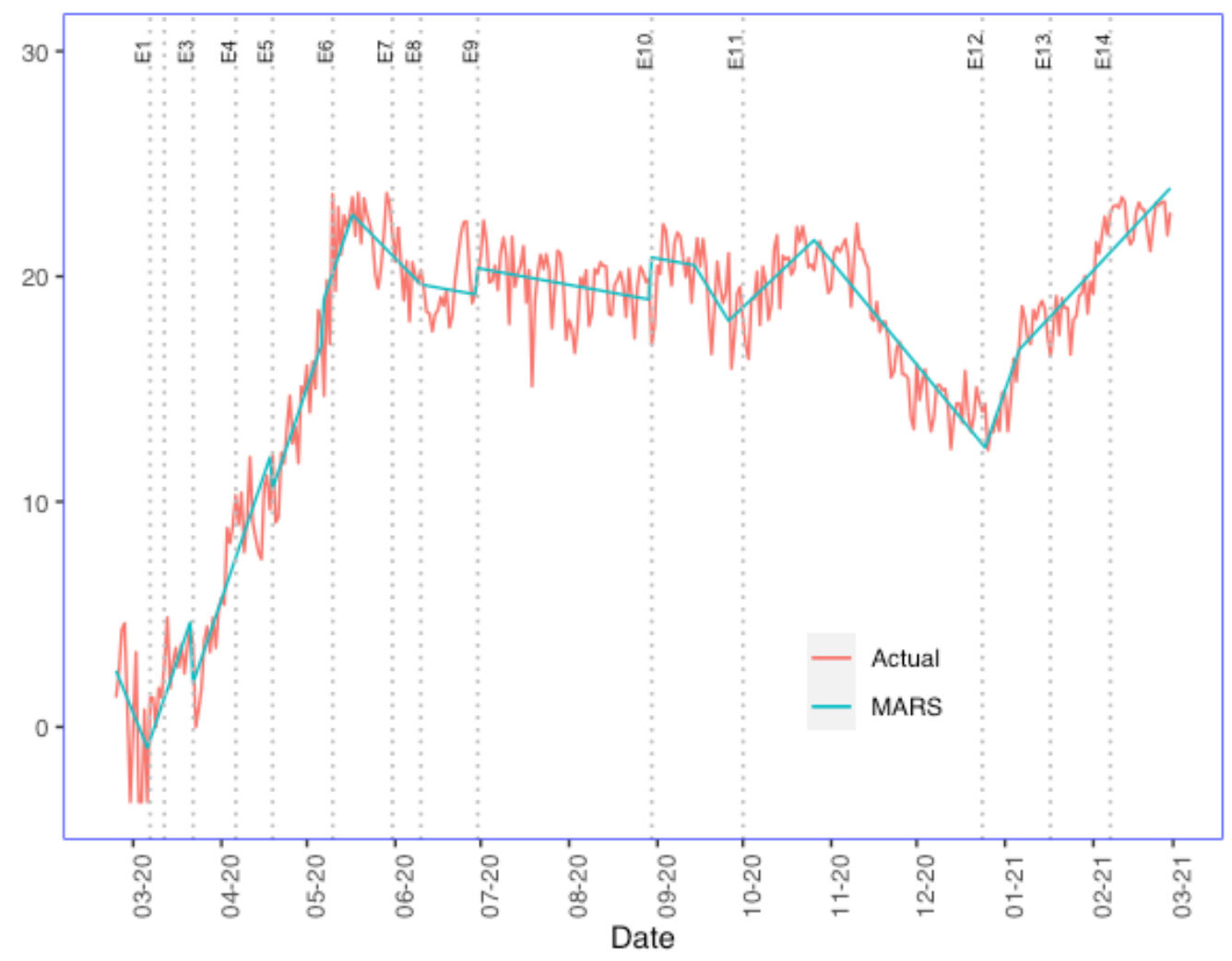

The model GCV, generalized $\mathrm{R}^{2}$ (GRSq), and $\mathrm{R}^{2}$ statistics were, respectively, 2.24, 0.95, and 0.94.

Figure 8 plots the actual values of the time series against the MARS estimated values. Remember that a $\mathrm{BF} \max (0, \mathrm{x}-\mathrm{c})($ or $\max [0, \mathrm{c}-\mathrm{x}])$ is defined as the maximum value of two elements, 0 and $\mathrm{x}-\mathrm{c}$, where $\mathrm{c}$ is a breakpoint. It has no effect on $\mathrm{Y}$ unless $\mathrm{X}-\mathrm{c}$ is positive. In this sense, $\max (0$, day 12$)$ became positive after the 12th day. A breakpoint at day 84 is indicated by the model terms 0.312 $\max (0$, day 84$)$ and $-0.812 \max (0$, day 84$)$. By that point, essential interventions E1-E6 had occurred, which might explain the decline in the curve after this point. The term $0.106 \max (0$, day 108) increased the curve after breakpoint 108. This happened about two weeks after E7 was implemented, when the total lockdown was changed to a partial curfew. The curve rose after breakpoint 204 and then fell after breakpoint 216, which occurred about two weeks after E10, when the partial curfew was lifted, and normal life resumed with restrictions. After breakpoint 246 , the term $-0.273 \max (0$, day 246$)$ significantly decreased the curve. This occurred after at least 11 interventions had been implemented. The contribution of the terms of the last two breakpoints increased the curve at day 306 and decreased the curve at day 318 . The latter occurred approximately two weeks after the vaccination campaign began. We also noticed that the most influential breakpoints in terms of decreasing the curve occurred on May 17 and October 26, 2020.

Figure 9 depicts a partial dependence plot for each predictor individually while keeping the other predictors at fixed levels. This will show the effect of that specific predictor on the model prediction. We used the "plotmo" package, which also allows us to visualize the effect of Y across the range of two predictors at the same time if interaction exists. The individual plots showed that our model found one knot in each of the interventions (E5, E9, and curfew) that provided the best fit, in addition 

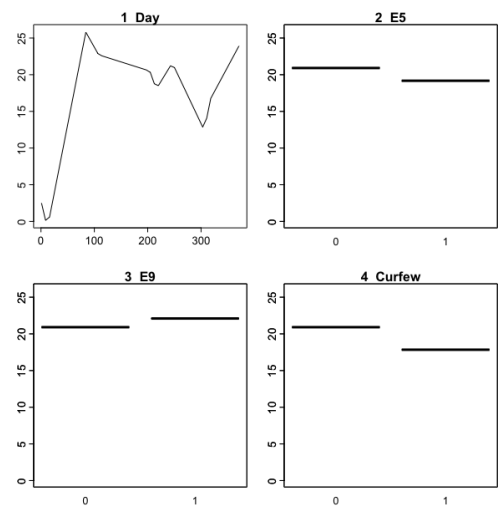

to the breakpoints in the time trend (day) predictor. Curfew activation (at level 1) results in a greater marginal decrease in Cases. Similarly, there is a slight decrease in the curve for E5 (evacuating citizens). Citizens were subjected to a 14-day special quarantine regardless of the results of PCR tests. When E9 (partial opening for workplaces and malls) was activated, there was a marginal increase.

\section{CONCLUSIONS}

The effect of a nonpharmaceutical intervention is considered in a novel application of intervention analysis to model the number of infected COVID-19 cases in Kuwait between February 24, 2020, and February 28, 2021. Our goal in this work was to investigate and quantify the effects of nonpharmaceutical interventions carried out in Kuwait during the pandemic period under study. To achieve this goal, two modeling procedures were used. The first method was to use intervention analysis with Arima modeling by fitting a structural break variable to the time series that reflected the major change points. The variable was then induced in the Arima model to obtain the best fit. The second method was to employ the MARS model, which included both quantitative (day) and qualitative (interventions) predictors. The mixed model's BFs are expressed differently for each type of variable. The nonparametric MARS method has the advantage of not requiring any prior assumptions about the statistical distribution of the data. Furthermore, MARS was not adversely affected by data collinearity, which is a common problem in interventional analysis. Also, its functions are clear and transparent, particularly when compared to other methods.

The main findings of our interventional analysis using $\operatorname{ARIMA}(5,2,1)$ showed a significant multiple-level shift caused by structural breaks with three breakpoints. The first occurred between days 59 and 61, indicating a 95\% confidence interval increase in the mean number of cases between $(584,639)$. Although the five interventions E1-E5 were already in place, this was the start of the pandemic's first wave. The second breakpoint occurred between days 258 and 264, reproducing a $95 \%$ confidence interval decrease in the mean number of cases between $(272,365)$. Following the implementation of the 11th intervention E11, there is a significant decrease in the mean number of cases. This model's last breakpoint occurred between days 313 and 319, resulting in an average increase in the mean number of cases between $(305,451)$ with a $95 \%$ confidence level. This was at the start of the pandemic's second wave, which occurred after increasing commercial flights (E13) from certain countries with a high pandemic rate.

The first three BFs in the MARS model correspond to binary interventions E5, E9, and curfew. As the curfew is activated, there is a greater marginal decrease in Cases. Similarly, there is a slight 
decrease in the curve for E5 (evacuating citizens). It should be noted that citizens were subjected to a 14-day special quarantine regardless of the PCR test results. When E9 (partial opening of workplaces and malls) was activated, there was a marginal increase. The remaining nine BFs involved time trend breakpoints or knots at days 12,84, 108, 204, 216, 246, 306, and 318. The two most influential breakpoints occurred at days 84 (May 17, 2020; E1-E6 were implemented) and 246 (October 26, 2020; post E11), where the cases were decreased in both incidences. Although the ARIMA $(5,1,2)$ model's metrics (RMSE $=1.39$ and MAE=1.03) were slightly lower than those of the MARS model $(\mathrm{RMSE}=1.48$ and $\mathrm{MAE}=1.17)$, the latter offers a more precise interpretation of the intervention's effects.

The findings of this research are generally in line with those of previous studies in terms of reducing the spread of the disease by implementing lockdown interventions. The impact of opening interventions differs from one country to another based on the effectiveness of and adherence to the precautionary measures through these openings.

The only pharmaceutical intervention in this study is vaccination (E12), which began in late December 2020. However, it is difficult to see the effect of the binary variable E12 within two months. To describe the effect of this pharmaceutical intervention, a quantitative measure of vaccination would be more appropriate.

\section{DATA/CODE AVAILABILITY STATEMENT}

Data and R codes for this study are available with the first author. 


\section{REFERENCES}

Al Dallal, A., Al Dallal, J. A., Ashqar, M., \& Al-Anzi, B. (2021). Trajectory Analysis of the Coronavirus Pandemic and the Impact of Precautionary Measures in the Kingdom of Bahrain. Arab Journal of Basic and Applied Sciences, 28(1), 135-144. doi:10.1080/25765299.2021.1886390

Al-Rousan, N., \& Al-Najjar, H. (2020). The impact of Chinese government plans on coronavirus CoVID-19 spreading and its association with weather variables in 30 Chinese provinces: Investigation. European Review for Medical and Pharmacological Sciences, 24(8), 4565-4571. PMID:32373996

Almeshal, A., Almazrouee, A., Alenizi, M., \& Alhajeri, S. (2020). Forecasting the spread of COVID-19 in Kuwait using compartmental and logistic regression models. Applied Sciences (Basel, Switzerland), 10(10), 1-18. doi:10.3390/app10103402

Bernal, J., Cummins, S., \& Gasparrini, A. (2017). Interrupted time series regression for the evaluation of public health interventions: A tutorial. International Journal of Epidemiology, 46(1), 348-355. PMID:27283160

Boehmke, B., \& Greenwell, B. (2019). Hands-On Machine Learning with R. Chapman and Hall/CRC. doi:10.1201/9780367816377

Cucinotta, D., \& Vanelli, M. (2020). WHO Declares COVID-19 a Pandemic. Acta Biomedica, 91(1), 157-160. PMID:32191675

Dalpiaz, D. (2020, October 30). Applied Statistics with R - David Dalpiaz. Retrieved April 23, 2021, from http:// daviddalpiaz.github.io/appliedstats/

Dowdy, D., \& D’Souza, G. (2020, August 10). Johns Hopkins Bloomberg School of Public Health. Johns Hopkins University. Retrieved May 10, 2021, from https://www.jhsph.edu/covid-19/articles/covid-19-testingunderstanding-the-percent-positive.html

Ferguson, N. M.-G., Imai, N., Ainslie, K., Baguelin, M., Bhatia, S., Boonyasiri, A., . . W. (2020). impact of non-pharmaceutical interventions (NPIs) to reduce COVID- 19 mortality and healthcare demand (Imperial College COVID-19 Response Team. London, UK: Imperial College London, MRC Centre for Global Infectious Disease Analysis. Retrieved from https://www.imperial.ac.uk/media/imperial-college/medicine/sph/ide/gidafellowships/Imperial-College-CO

Friedman, J. (1991). Multivariate Adaptive Regression Splines. Annals of Statistics, 19, 1-141.

Funk, S., Ciglenecki, I., Tiffany, A., Gignoux, E., Camacho, A., \& Eggo, R. (2017). The impact of control strategies and behavioural changes on the elimination of Ebola from Lofa County, Liberia. Philosophical Transactions of the Royal Society B: Biological Sciences, 372(1721), 1-11.

Garziano, G. (2017, October 7). Arima models and Intervention Analysis. R-Bloggers. Retrieved March 24, 2021, from https://www.r-bloggers.com/2017/10/arima-models-and-intervention-analysis/

Hsieh, C.-C., Lin, C.-H., Wang, W., Pauleen, D., \& Chen, J. (2020). The outcome and implications of public precautionary measures in Taiwan-declining respiratory disease cases in the COVID-19 pandemic. International Journal of Environmental Research and Public Health, 17(13), 1-10. doi:10.3390/ijerph17134877 PMID:32640752

Leach, S. (2008). Haemorrhagic fevers in Africa: narratives, politics and pathways of disease and response. STEPS Working Paper 14. STEPS Centre. Retrieved from https://steps-centre.org/publication/haemorrhagicfevers-in-africanarratives-politics-and-pathways-of-disease-and-response/

Lee, T., Chiu, C., Chou, Y., \& Lu, C. (2006). Mining the customer credit using classification and regression tree and multivariate adaptive regression splines. Computational Statistics \& Data Analysis, 50(4), 1113-1130. doi:10.1016/j.csda.2004.11.006

McKibbin, W., \& Fernando, R. (2020). The Global Macroeconomic Impacts of COVID-19- Seven Scenarios. Centre for Applied Macroeconomic Analysis.

Ministry of Health Kuwait. (2021). Retrieved March 1, 2021, from https://www.moh.gov.kw/ 
OEB. (2017, December 29). Regression with caret - multivariate adaptive regression splines (MARS). Retrieved August 11, 2020, from https://rstudio-pubs-static.s3.amazonaws.com/376822_e229bf5a5d574f7c93e12cb524f726f1.html

PennState. (2021). Applied Time Series Analysis. Retrieved March 24, 2021, from https://online.stat.psu.edu/ stat510/lesson/9/9.2

Policy Brief. (2020). A policy framework for tackling the economic and social impact of the COVID-19 crisis. International Labour Organization.

Sheskin, D. (2011). Handbook of Parametric and Nonparametric Statistical Procedures. Chapman \& Hall/CRC.

Tandon, H., Ranjan, P., Chakraborty, T., \& Suhag, V. (2020). Coronavirus (COVID-19): ARIMA based timeseries analysis to forecast near future. Academic Press.

Turner, S., Karahalios, A., Forbes, A., Taljaard, M., Grimshaw, J., Cheng, A., Bero, L., \& McKenzie, J. (2020). Design characteristics and statistical methods used in interrupted time series studies evaluating public health interventions: A review. Journal of Clinical Epidemiology, 122, 11. doi:10.1016/j.jclinepi.2020.02.006 PMID:32109503

Vokó, Z., \& Pitter, J. G. (2020). The effect of social distance measures on COVID-19 epidemics in Europe: An interrupted time series analysis. GeroScience, 42(4), 1075-1082. doi:10.1007/s11357-020-00205-0 PMID:32529592

WHO Coronavirus Disease (COVID-19) Dashboard. (2021). Retrieved May 20, 2021, from https://covid19. who.int

World Health Organization, Food and Agriculture Organization of the United Nations, \& United Nations Children's Fund (UNICEF). (2012). Communication for Behavioural Impact (COMBI): A toolkit for behavioural and social communication in outbreak response. Retrieved from https://www.who.int/ihr/publications/combi_ toolkit_outbreaks/en/

Zhu, N., Zhang, D., Wang, W., Li, X., Yang, B., Song, J., Zhao, X., Huang, B., Shi, W., Lu, R., Niu, P., Zhan, F., Ma, X., Wang, D., Xu, W., Wu, G., Gao, G. F., \& Tan, W. (2020). A Novel Coronavirus from Patients with Pneumonia in China, 2019. The New England Journal of Medicine, 382(8), 727-733. doi:10.1056/ NEJMoa2001017 PMID:31978945

Sana BuHamra is an Associate Professor at the Information Science Department, Kuwait University (KU). She earned her master's in applied Statistics from Ohio State University, and PhD in Statistics from University of Wyoming, USA. She was the founding Dean of College for Women, which is now known as the College of Life Sciences. Throughout her career, she received numerous honors and awards. Sana is interested in data mining, data analysis, health informatics as well as nonparametric testing, environmental statistics, and questionnaire design.

Jehad Al Dallal is a Professor at Computer Science Department, Gulf University for Science and Technology. He received his PhD in Computer Science from the University of Alberta in Canada and was granted the award for best PhD researcher. Prof. Al Dallal has completed many research projects in the areas of software testing, software metrics, software refactoring, and communication protocols. In addition, he has published more than 100 papers in conference proceedings and ACM, IEEE, IET, Elsevier, Wiley, and other journals. Prof. AI Dallal was involved in developing more than 20 software systems. He also served as a technical committee member of several international conferences and as an associate editor for several refereed journals. 